\title{
The screening of multi-drug resistance (MDR) susceptibilities of Staphylococcus aureus and Staphylococcus epidermidis to methicillin and vancomycin in teaching hospitals in Nigeria Yah SC ${ }^{2}$, Enabulele $\mathrm{IO}^{1}$ and Eghafona $\mathrm{NO}^{1}$
}

\begin{abstract}
Background: In Nigeria, the widespread use of antibiotics had led to high levels of resistance among bacterial isolates from patients with nosocomial infections. This had led to prolonged hospital stay and antibiotic therapy, especially $\beta$-lactam antibiotics that predispose patients to acquisition of methicillin -resistant Staph. aureus (MRSA) and coagulase negative resistant staphylococci.
\end{abstract}

Objective: to evaluate the resistant pattern of multi-drug resistant strains of 80 clinical Staph. aureus, 22 environmental Staph. aureus, 30 clinical Staph. epidermidis and 12 environmental Staph. epidermidis to methicillin and vancomycin from teaching hospitals in Nigeria.

Material and Methods: The Staphylococcus species were identified and confirmed by gram-positive positive reaction, tested for mannitol salt fermentation and DNase production. The organisms were confirmed to be Staph. aureus and Staph epidermidis by the tube coagulase test. The antibiotics susceptibility patterns were determined both by overnight broth-microdilution and agar disk diffusion methods.

Results: The isolates were resistant to ampicillin, followed by penicillin, tetracycline, erythromycin and gentamicin but to a lesser extent were sensitive to ciprofloxacin. All the multi-drug resistant (MDR) Staphylococcus species were $100 \%$ sensitive to vancomycin and methicillin with a minimum inhibition concentration (MIC) breakpoint $<4 \mu \mathrm{g} / \mathrm{ml}$ to vancomycin and MIC $<$ $5 \mu \mathrm{g} / \mathrm{ml}$ to methicillin on Mueller Hinton agar supplemented with $2 \% \mathrm{NaCl}$.

Conclusion: The results indicated that methicillin and vancomycin are still very potent antibiotics against staphylococcal infections in Nigeria.

Key Words: MDR Staphylococcus, methicillin and vancomycin.

\section{Introduction}

In Nigeria, the widespread use of antibiotics had led to high levels of resistance among bacterial isolates from patients with nosocomial infections $19-20,15$. This had led to prolonged hospital stay and antibiotic therapy, especially $\beta$ lactam antibiotics that predispose patients to acquisition of methicillin -resistant Staph. aureus (MRSA) and coagulase negative resistant staphylococci. Methicillin resistant strains that emerged by late 1980 s have become increasingly present as nosocomial pathogens. The medical community was again relieved when vancomycin a glycoprotein was discovered that added effective therapy to all strains of methicillin resistant Staph. aureus. Nevertheless vancomycin resistant strains of coagulase-negative staphylococci were also a cause of concern ${ }^{16,7,14,18}$. Added to these concerns were observations that vancomycin resistant enterococci isolates or epidemics in some U.S. hospitals were becoming increasingly prevalent in critical care units ${ }^{5,4}$ and high level vancomycin resistance were experimentally transferred from Enterococcus

2- Department of Microbiology, College of Science and Technology, Covenant University, Km 10 Idiroko Road, Ota, Ogun State, Nigeria.

1- Department of Microbiology, Faculty of Life Sciences, University of Benin, Benin City, Nigeria.

Correspondance, Dr Yah Clarence S. Nigeria, Email: yahclar@yahoo.com Phone +2348053336108 or $+2348063418265$ faecalis to Staph. aureus in both in- vitro and in vivo- models ${ }^{4,7}$. Strains of Staph. aureus and gram negative organisms resistant to vancomycin and other antimicrobial agents including quinolones are endemic already in numerous hospitals and health care institutions leaving only a few effective and costly antimicrobials for the treatment of patients infected with these pathogens ${ }^{8}$. In Nigeria, there has been a recent increase in resistant to gentamicin and variable susceptibility to other non-B-lactam antibiotics, namely tetracycline, trimethoprim, erythromycin and ciprofloxacin ${ }^{1,22,24}$. In this study we investigated both the broth-micro-dilution and agar disk diffusion methods on multi-drug resistant on both hospital environment and long term clinical isolates of Staph. aureus and Staph. epidermidis from some selected teaching hospitals in Nigeria to ascertain their level of resistance to methicillin and vancomycin.

\section{Methods}

Bacterial strains and selection of isolates for analysis: One hundred and forty four multi-drug resistant Staph. aureus and Staph. epidermidis from some selected teaching hospitals in Nigeria were obtained and examined for their antibiotics susceptibility profiles to methicillin and vancomycin. These isolates include 80 multi-drug resistant clinical Staph. aureus strains, 22 multidrug resistant environmental Staph. aureus strains and 30 multi-drug resistant clinical Staph. epidermidis strains and 12 multi-drug resistant environmental Staph. epidermidis strains. The clinical isolates were obtained randomly from routine specimens from different infected sites 
(urine, wounds, and diarrheal stool) of prolonged hospitalized patients between May 2003 and October 2004. The environmental isolates were obtained from the teaching hospitals wards (air) by the suck-let sampler method. The teaching hospitals were; University of Benin Teaching Hospital (UBTH), Benin City, Edo StateSouthern Region of Nigeria; Nnamdi Azikiwe Teaching Hospital (NAUTH), Nnewi, Anambra State - Eastern Region of Nigeria; Ahmadu Bello University Teaching Hospital (ABUTH), Zaria Kaduna State - Northern Region of Nigeria and University College Hospital (UCH), Madan, Oyo State- Western Region of Nigeria.

Bacterend identification: All Staph. aureus and Staph. epidermidis strains were identified primarily by routine laboratory procedures ${ }^{9}$ and confirmed to be Staph. aureus and Staph. epidermidis by gram-positive cocci norphology, catalase-positive reaction were tested for mannitol salt fermentation (Oxoid, Melbourne, Australia) and DNase production on agar plates (Oxoid CM321). Clumping factor was detected by using rabbit plasma. Organisms were confirmed to be Staph. aureus and Staph. epidermidis by the tube coagulase test

Antibiotic Sensitivity Testing: The antibiotics susceptibility patterns were determined both by overnight broth-micro-dilution and agar disk diffusion methods as recommended by Bauer et $a^{12}$ and National Committee for Clinical Laboratory Standard ${ }^{17}$ using Oxoid. Mueller Hinton agar (Difco Laboratories, Detroit, Mich). The following antibiotics were used to screen for the resistance of the isolates; ampicillin (AM) $30 \mu \mathrm{g}$, gentamicin (GN) 10 $\mathrm{gg}$, tetracycline (TE) $30 \mu \mathrm{g}$, ciprofloxacin (CIP) $5 \mu \mathrm{g}$, erythromycin (E) $10 \mu g$, Penicillin (PN) 30ug (Optun Laboratories Nig Lid., Nigeria), methicillin $5 \mu \mathrm{g}$ (Bristol Meyers Squib) and vancomycin (VAN) $5 \mu \mathrm{g}$ (Mayne Pharma Warwickshire -UK). The inocula were prepared directly from an over night agar plate. Investigation of bactericidal activity were done by measuring the zone of inhibition with standard measuring procedures according to NCCLS, ${ }^{17}$ after incubation at $30-350 \mathrm{C}$ for 24 hours. Staphylococcus strains that showed resistance to three or more classes of antibiotics were titled as 10 multi drug resistant (MDR), and were further preserved for other analyses. The fully sensitive strains of the organisms were discarded.

Agar Dilution tests of methicillin and vancomycin: The minimum inhibition concentration (MIC) of methicillin (MET) $500 \mathrm{mg}$
(Bristol Meyers Squib Hampshire- England) and vancomycin (VAN) 500mg (Mayne Pharma Warwickshire -UK) was determined by agar dilution method, according to the guidelines of NCCLS. ${ }^{17}$ Colonies of each strain from an over night growth were transferred to sterile saline. The suspension were adjusted to a $0.5 \mathrm{McFarland}$ standard, diluted 1:10, and inoculated on Mueller Hinton agar (Difco Laboratories, Detroit, Mich) plates supplemented with $2 \% \mathrm{NaCl}$ wt/vol. The plates were incubated at $30-350 \mathrm{C}$ for 24 hours.

mesulls

The comparative antibiotic susceptibility profiles of the 80 multi-drug resistant clinical Staph. aureus, 22 multi-drug resistant onvironmental Staphylococcus aureus and 30 multi-drug resistant clinical Staph. epidermidis and 12 multi-drug resistant environmental Staph. epidermidis were shown in Tables 3 a to $3 d$. All the MDR isolates were sensitive to methicillin and vancomycin, but were resistant to ampicillin, followed by penicillin, tetracycline, erythromycin, gentamicin and ciprofloxacin. The results showed that both isolates were highly resistant to ampicillin and penicillin from all the teaching hospitals with resistant ranged of $40 \%$ to $71 \%$. The isolates also had a resistant range of $00 \%$ to $17 \%$ to ciprofloxacin, although environmental Staph. epidermidis were $00 \%$ resistant to ciprofloxacin. Only Staph. epidermidis from UBTH as shown in Table 1 had a resistance of $17 \%$ to ciprotloxacin. The resistance patern varied among the gentamicin, erythromycin and tetracycline as shown in Tables $3 a$ to $3 b$. The minimum inhibitory concentration (MIC) range was $0.5 \mu \mathrm{g} / \mathrm{ml}$ to $5 \mu \mathrm{g} / \mathrm{ml}$ with a MIC break point of $<4 \mu \mathrm{g} / \mathrm{ml}$ for both isolates as shown in Tables $3 c$ and $3 d$. All the 144 MDR Staph. aureus and Stoph. epidermidis were considered to be susceptible to methicillin and vancomycin according to published NCCLS guidelines. None of the isolates had a $\mathrm{MIC}>5 \mu \mathrm{g} / \mathrm{ml}$

Discussion

The 144 MDR isolates in this report were described as sensitive isolates to methicillin and vancomycin. This contradicts other reports from Nigeria and some other African countries." 15. Since all the strains were sensitive to methicillin and vancomycin, the study therefore suggested that none of the strains contained van $A$ or mecA genes respectively. The rate of resistance continues to reduce from the earlier reports ${ }^{15}$, the 
The screening of multi-drug resistance in teaching hospitals in Nigeria, Yah SC et al

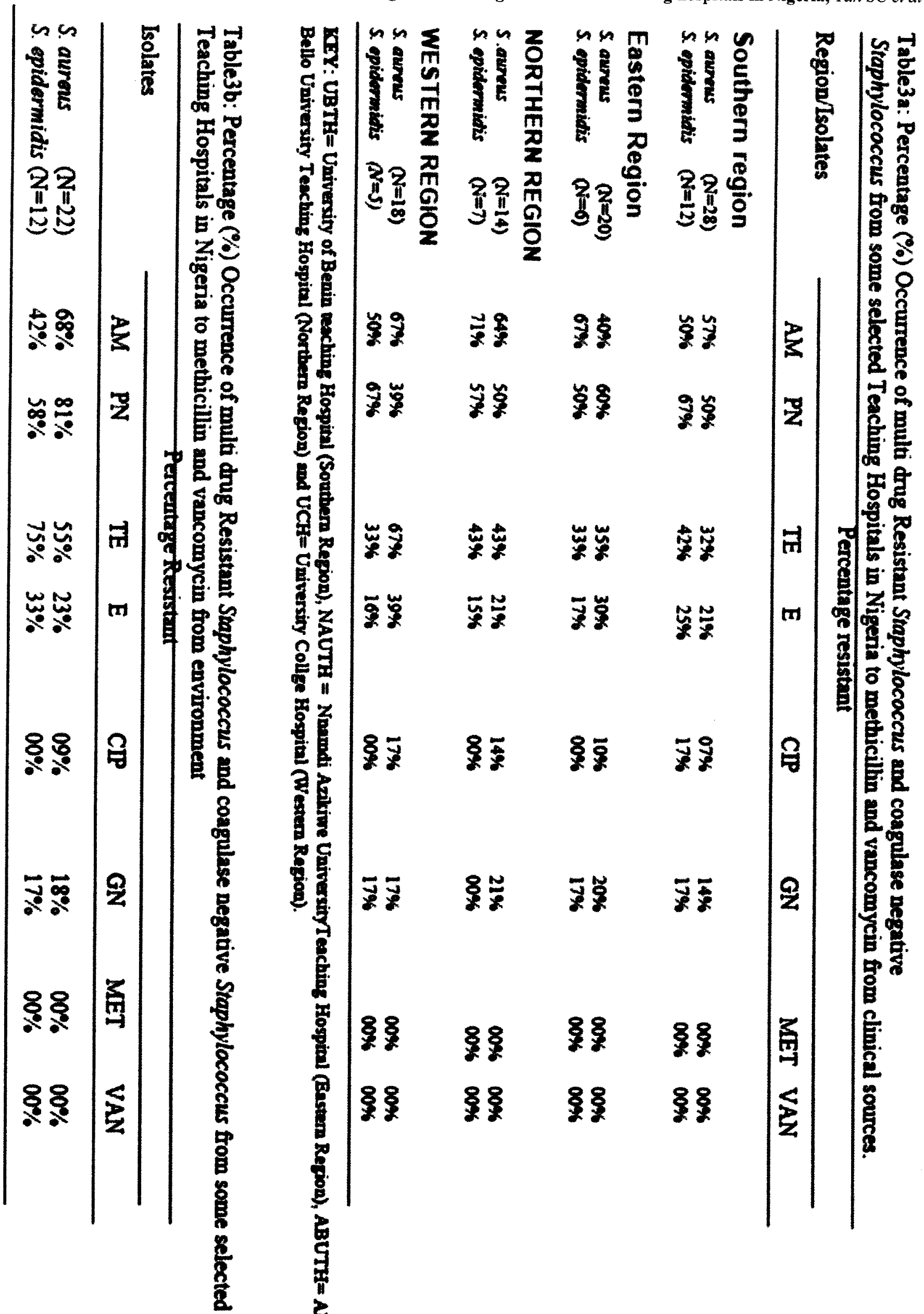




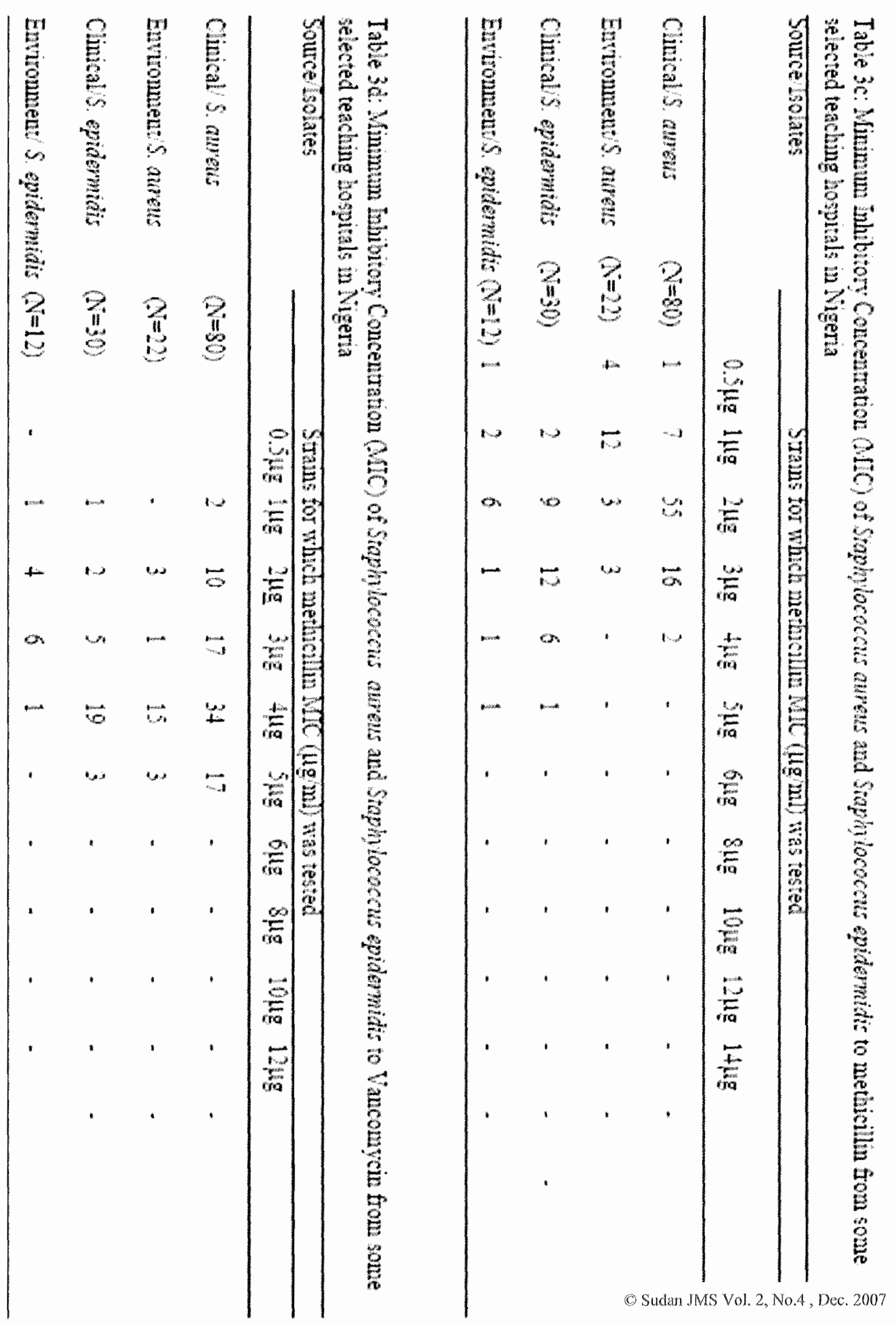


isolates according to the present study were all sensitive to both methicillin and vancomycin. The current studies also found that methicillin, oxacillin and vancomycin were not at all among the commonly prescribed antibiotics in teaching hospital in Nigeria ${ }^{25}$. However, cloxacillin with similar mode of action was very rare in circulation as compared to commonly prescribed ampicillin, penicillin, aminoglycosides and quinolones ${ }^{23}$.

Despite the fact that a $\mathrm{MIC}=4 \mu \mathrm{g} / \mathrm{mL}$ was defined as susceptible by NCCLS standards ${ }^{17}$, it is still considered to be at the borderline of resistance. S. aureus strains that are methicillin or oxacillin resistant and have a MIC of vancomycin $\geq 4 \mu \mathrm{g} / \mathrm{mL}$ should be suspected for decreased susceptibility to vancomycin and should be considered for additional testing strategies because of the possible sub-population heterogeneity of S. aureus isolates with these MIC results ${ }^{6,22}$. Our results indicate that methicillin and vancomycin are still very potent antibiotics against Staph. aureus and Staph. epidermidis infections. According to Jan et $\mathrm{al}^{10}$, strains of MRSA with reduced susceptibility to vancomycin were isolated in Japan in 1997 and have since been described in the United States, France, Hong Kong, China, and Korea. Their findings as well had no strains of vancomycin intermediate Staph. aureus despite having three sites in Japan, consistent with the suggestion that these strains are still relatively rare. The isolation of these strains in an area of high endemicity indicates the need for continuous surveillance of antibiotic resistance of Staphylococcus species and the rationalization of antibiotic in clinical set up.

\section{Conclusion}

The results indicated that methicillin and vancomycin are still very potent antibiotics against Staph. aureus and Staph. epidermidis infections. Therefore the cry of methicillin-resistant Staph. aureus (MRSA) that was first identified in the United Kingdom in 196111 and since then assumed increasing importance internationally as a cause of both nosocomial and community-acquired infections should not be the case in Nigeria for now.

\section{Acknowledgement}

We will like to thank Dr Oranusi Solomon of Department of Microbiology ABU Zaria, Rauol Ndasi of the Department of Histopathology ABUTH Zaria, Dr Joseph Forbi of the Department of Virology UCH Ibadan, Tchuendem Fopoussi of the Department of Histopathology NAUTH Nnewi and Staff of the Department of Medical Microbiology especially Dr E.O.Yusuf UBTH Benin City for their contributions.

\section{References}

1. Ahonkhai, I., and Monye, A.C.. In-Vitro effect of ciprofloxacin on methicillin resistant isolates of Staphylococcus aureus. Journal of Medical Laboratory Sciences 1996; 5: 69-72.

2. Bauer AW, Kirby WM, Sherris JC. Antibiotics susceptibility testing by a standardized single disk method. AmJ Clin Patho 1997; 45: 493-496.

3. Beth, E. D., ANn M. E. ANd Richard A. V. Vancomycin AND OXACILLIN SYNERGY FOR METHICILLIN-RESISTANT STAPHYLOCOCCI. ANTIMICROBIAL AGENTS AND CHEMOTHERAPY2000; 44(5): 1394- 1396.

4. Coronodo BG, Edwards JR and Culver DH. Ciprofloxacin resistance among nosocomial Pseudomonas aeruginosa and Staphylococcus aureus in the United States. Infect control. Hospital Epidemol 1995; 16: 71-75.

5. Doern GV, Brueggaman A and Hoolay HP. Antimicrobial resistance of Streptococcus pneumoniae recovered from out patient in the U.S.A.during the writer months of 19941995. Results of a so center national surveillance study. Antimicrobiols Agents Chemothes 1996; 40:1208-1213.

6. Fridkin SK. Vancomycin-intermediate and resistant Staphylococcus aureus; what the infection disease specialist needs to know. Clin Infect Dis 2001;3 2:108-15.

7. Goldman DA, Weintein RA, Wenkel RP et al. Strategies to prevent and control the emergence and spread of antimicrobial -resistance microorganisms in hospital: A Challenge to hospital leadership. JAMA 1996; 275: 23440 .

8. Goossens H. MYSTIC program: summary of European data from 1997 to 2000. Diagn Microbiol Infect Dis 2001; 41:183-189.

9. Hackbarth C J and Chambers H F. Methicillin-resistant staphylococci: detection methods and treatment of infections. Antimicrobial Agents and Chemotherapy 1989; 33: 995-9.

10. Jan M B, John D T and SENTRY APAC Participants. High Prevalence of Oxacillin-Resistant Staphylococcus aureus Isolates from Hospitalized Patients in Asia-Pacific and South Africa: Results from SENTRY Antimicrobial Surveillance Program, 1998-1999. Antimicrob Agents Chemother2002; 46(3): 879-881

11. Jevons M P. "Celbenin"-resistant staphylococci. BM J $1961 ; 1: 124-125$.

12.John M, Jason W, Barbara $R$ et al. Detection and expression of methicillin/oxacillin resistance in multidrugresistant and non-multidrug-resistant Staphylococcus aureus in Central Sydney, Australia. Journal of Antimicrobial Chemotherapy2002; 49: 793-801.

13.Jones RN, Mathai D and Rhomberg PR A comparison of the antimicrobial activity of meropenem and selected broad-spectrum antimicrobials tested against multi-drug resistant Gram-negative bacilli including bacteraemic Salmonella spp:: initial studies for the MYSTIC programme in India. Int $J$ Antimicrob Agents 2002; $20: 426-431$.

14. Jones RR, Resistance patterns among nosocomial pathogens. Trends over the past few years. Chest 2001;119: 397S-404S.

15. Kesah C, Redjeb SB, Odugbemi TO et al. Prevalence of methicillin resistant Staphylococcus aureus in eight African countries and Malta. Clin Microbio Infect 2003;9: 153-156.

16.Low DE and Scheild WM. Strategies for stemming the tide of antimicrobial resistance. JAMA 1988; 279: 394395 . 
17. National Committee for clinical laboratory standards. 1997. Methods for Dilution Antimicrobial Susceptibility Test for Bacteria that Grow aerobically.4th Edition. Approved Standard M7-A4. National Committee for clinical laboratory standards, Wayne $\mathrm{Pa}$

18. O'Brien FG, Lim TT, Chong FN et al. Diversity among community isolates of Methicillin resistant Staphylococcus aureus in Australia. I Clin Microbiol 2004; 42: 3185 3190.

19. Obaseki-Ebor EE, Akerele JO and Ebea PO. A survey of antibiotic outpatient prescribing and antibiotic selfmedication. J Antimicrob Chemother 1987; 20: 759-763.

20. Okeke I, Lamikanra A. Quality and bioavailability of tetracycline capsules in a Nigerian semi-urban community. Intern J Antimicrobial Agents 1995; 5:245-50.

21. Paterson DL, Wen-Chien $K$ and Von Gottberg $A$. Outcome of cephalosporin treatment for serious infections due to apparently susceptible organisms producing extended-spectrum $\beta$-lactamases: implications for the clinical microbiology laboratory. J Clin Microbiol 2001 39: 2206-2212.

22. Ronald NJ, Helio SS, Thomas RF et al. Selection of a surrogate agent (Vancomycin and teicoplanin) for initial susceptibility testing of Dalbavancin: results from international antimicrobial surveillance program. Journal of Clinical Microbiology2006; 44: 2622-2625.

23. Rotimi VO, Oredanjo OA, Banjo TO et al Occurrence and antibiotics susceptibility profiles in methicillin resistant Staphylococcus aureus in Lagos, university Teaching Hospital. Cen Afric J Med 1987; 33: 95-9

24. Tenover FC, Lancaster MV, Hill BC et al Characterization of staphylococci with reduced susceptibilities to vancomycin and other glycopeptides. Clin Microbiol 1998;36:1020-7.

25. Yah SC, Enabulele IO and Eghafona NO. Bacteriological studies on infected Kerosene burn wounds in Benin City, Nigeria. Journal of Biomedical Investigation (JBI) 2004; 2(1): 4-9. 